\title{
With the Basis of the Improved SAC Code of OCDMA Wavelength Division Passive Network Security Research
}

\author{
Lin Yang \\ Sanya University, Sanya Hainan 572022, China
}

\begin{abstract}
This paper will improve the spectral amplitude coding is used for OCDMA wavelength division multiplexing passive optical network system. Improved the system bit error rate of less than $10^{-10}$, multiple access interference suppressed to some extent, the performance of the transmission network stable and less volatile, strong coding scheme of randomness, invasion of increased difficulty, confidentiality system have the corresponding increase.
\end{abstract}

Keywords-SAC; OCSMA; WDM; MQC

\section{INTRODUCTION}

In recent years, researchers used OCDMA technique wavelength division multiplexing passive optical network system, In OCDMA passive WDM network system encoding has a very important position, so choose encoding must meet the needs and characteristics of the transmission system. This requires all aspects of the system are taken into account to choose the most optimal solution or compromise way ${ }^{[1]}$.

Advances in technology OCDMA address code construction codec design for OCDMA WDM passive network technology implementation provides favorable conditions. In this paper, an improved SAC improve user capacity and improve transmission quality and safety performance. In design, each user has a unique address code can identify, extract relations with features matching the optical signal through a corresponding optical device and associated optical signal processing ,thereby inhibiting or reducing the level of mutual interference between different users $^{[2]}$.

\section{OCDMA-BASED WDM PASSIVE NETWORK SYSTEM}

OCDMA WDM passive network system composed by the OLT, RN, ONU and AWG. In the upstream direction systems generally use star structure, adopting WDMA different wavelength of ONU settlement channel contention, CDMA was adopted to realize multiple access of the same wavelength ONU. On the transmission network, different users of pseudo random CDMA overlay into corresponding transmission signals sent by radio, having attained the $\mathrm{RN}$, assigned to the corresponding terminal node demodulation signal light. Judging by the demodulation of the signal correlation signal trade-offs, which would hold autocorrelation decoding, shielding cross-correlation signal decoding ${ }^{[3]}$.

It's very high for coding for OCDMA wavelength division directly influences the performance of passive network system.
Therefore the choice of coding is very important, coding in the choice of address code is a top priority. User address code consists of multiple code word, the number of user, the longer the length of the address, and need to have performance good autocorrelation and cross-correlation properties ${ }^{[4]}$. For strictly synchronous transmission system, user interference demodulation signal is in control, address code structure can simply the corresponding processing. The entire network synchronization issues need to be resolved ${ }^{[5]}$.

OCDMA wavelength division passive network system overall structure as shown in Figure I.

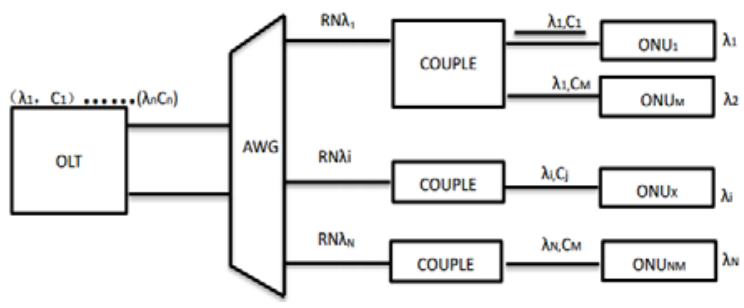

FIGURE I. GENERAL STRUCTURE OF OCDMA WAVELENGTH DIVISION PASSIVE NETWORK SYSTEM

System capacity $\mathrm{C}$ equals reuse wavelength number $\mathrm{N}$ and the product of the code word number $M$, namely $C=N \times M$. Each subsystem has a relatively independent a wavelength, OCDMA code word capacity determines the subsystem membrane size, so the same orthogonal OCDMA code word can be reused in different subsystem. Signal data from OLT side uses the direct modulation or modulation mode. OLT encapsulates many wavelengths of light within the coding and transceiver array. When the modulation signal data is loaded into the $\mathrm{N}$ light samples of the different carrier.

\section{SYSTEM ANALYSIS}

SAC is an important coding solution can be used in OCDMA optical networks. Adjusted MQC encoding is a typical SAC, due to its good automatic cross-correlation characteristic has been used as a regular code sequences. But in the OCDMA wavelength division, we can use good-correlation MQC encoding used in spectral amplitude coding. According to the MQC encoding attribute, assume that $p$ is a prime number, $F$ is code length, then $F=\mathrm{P}^{2}+\mathrm{P}$, but code weight $\omega=\mathrm{P}+1$. Two parts through balancing the probe signal, the final decision output light signal is ${ }^{[6]}$ : 


$$
\begin{gathered}
\sum_{i=1}^{F} C_{k}(i) C_{1}(i)=\left\{\begin{array}{l}
P=1, k=1 \\
1 \quad, k \neq 1
\end{array}\right. \\
\sum_{i=1}^{F} C_{k}(i) C_{l}(i)=\left\{\begin{array}{l}
0, k=1 \\
P, k \neq 1
\end{array}\right.
\end{gathered}
$$

Assume that light source spectrum density of unilateral is $\mathrm{G}(\mathrm{v})$, the receiver general equation for the signal is

$$
\begin{gathered}
G^{i}(v)=\frac{P_{e r}}{\Delta v} \sum_{n=1}^{N=P^{2}} b_{n} \sum_{i=1}^{F=P^{2}+P} C_{n}(i) \times \\
\left(\begin{array}{c}
u\left[v-v_{0}-\frac{\Delta v}{2 P^{2}}\left(-P^{2}+2 i-2\right)\right] \\
-u\left[v-v_{0}-\frac{\Delta v}{2 P^{2}}\left(-P^{2}+2 i\right)\right]
\end{array}\right)
\end{gathered}
$$

$P_{e r}$ is signal strength, suppose in the system power consumption is much less than $P_{e r}$, than light amplitude is $P_{e r} / \Delta v$, flat wavelength range, the non-polar light source, wavelength range within $v_{0} \pm \Delta v / 2 . b_{n}$ and $c_{n}$ is the $\mathrm{n}$ user bit sequence spread spectrum code sequence. $\mathrm{N}$ is the total number of users, $\mathrm{u}(\mathrm{v})$ is step function.

In an ideal world, suppose the related parameters in the light $\tau=0$, the receiving end receiver current is $i^{2}=$ $2 e I B+I^{2} B \tau_{c}+4 K_{b} T_{n} B / R_{L} . e$ is charge constant, $I$ is review current, $B$ is bandwidth, $K_{b}$ Boltzmann's constant, $T_{n}$ is absolute receiver noise temperature, $R_{L}$ is the receiver of the load resistance. Ideally, you can ignore other unpitched sound. According to the $G(v)$ general equation, can be obtained by light source spectrum density of unilateral coherence parameters $\tau_{c}=\int_{v=0}^{\infty} G^{2}(v) d v /\left[\int_{v=0}^{\infty} G(v) d v\right.$.

Ideally, users is synchronized, no considering the wastage of the system, $G_{1}^{2}(v)$ and $G_{2}^{2}(v)$ integral:

$$
\begin{aligned}
& \int_{0}^{\infty} G_{1}^{2}(v) d v \\
& =\frac{P_{e r}^{2}}{P^{2} \Delta v} \sum_{n=1}^{F}\left\{C_{1}(i)\left[\sum_{n=1}^{N} b_{n} c_{n}(i)\right]\left[\sum_{m=1}^{N} b_{m} c_{m}(i)\right]\right\} \\
& \int_{0}^{\infty} G_{2}^{2}(v) d v \\
& =\frac{P_{e r}^{2}}{F \Delta v} \sum_{n=1}^{F}\left\{C_{1}(i)\left[\sum_{n=1}^{N} b_{n} c_{n}(i)\right]\left[\sum_{m=1}^{N} b_{m} c_{m}(i)\right]\right\}
\end{aligned}
$$

We can through the photoelectric diode current to the different output to calculate the user needs to receive the signal average current value $\mathrm{I}, \mathrm{I}=\mathrm{I}=I_{1}-I_{2}=\zeta P_{e r} / P b_{1}$, $\zeta=\eta \mathrm{E} / \mathrm{H} V_{c}$ the response of the photodiode, $\eta$ is quantum efficiency, h is Planck's constant, $V c$ is the center of the original broadband optical pulse frequency. Considering some other related features, according to the variance of noise power formula, SNR value can be drawn from. The value and the response of the diode and current is proportional to the receiver, inversely proportional to the capacitance and resistance characteristics.

At present, the channel congestion have bigger influence on the security, so in the case of improve the utilization rate of channel can be used to improve the MQC code to realize the increase of channel use. The influence degree of the system by the intensity of noise also increases accordingly. The utilization rate of the system is close to $100 \%$. In the case of channel a gradual increase in the total, reduce the error code. After the improvement design MQC code, BER can use Gaussian approximation estimates, $B E R=1 / 2 \operatorname{erf} c \sqrt{S N R / 8}$. In the process of experiment, eavesdropper needs to detect the user signal of each pulse, detected the authorized user of the pulse code word probability depends on the amount of time detection scheme. Based on analysis of classic detection, if eavesdroppers to intercept a code word observation signal transmission by the time depends on the spectral amplitude coding optical encoding the length of the CDMA data bits. The general classification code word detection probability is

$$
P_{\text {corrent }}=\left[Q\left(\sqrt{\frac{2 E}{N_{0}}}, \sqrt{\frac{2 \gamma}{N_{0}}}\right)\right]^{\omega}\left[1-\exp \left(-\frac{\gamma}{N_{0}}\right)\right]^{(F-\omega)}
$$

$\mathrm{Q}$ is a common Marcum probability integral function, $E / N_{0}$ is the ratio pulse energy and noise power spectral density, $\gamma$ is detector threshold, $\mathrm{F}$ is code length, $\omega$ is code weight.

Based on the analysis of conventional malicious third party detection, in the code block spectral amplitude coding length coding OCDMA address data, so can be modified through the use of MQC code weight method to improve system security ${ }^{[7]}$. Through the simplified model to approximate deduction and calculation, an allowed within the scope of errors calculation results and the actual situation are appeared. While using the traditional nonpolar coding, but improve the OCDMA wavelength division passive network system resource utilization.

\section{CONCLUSION}

OCDMA system applications and access to the network when there are more advantages, become the most attractive technology. These advantages include the flexibility to assign users, asynchronous access, burst traffic, support for variable bit rates and security, and prevent unauthorized users from using it. OCDMA WDM passive network technology combines the dual advantages of OCDMA technology and WDM-PON technology, which is the future direction of optical access network, increases the system capacity, the security of the physical layer network and improves the function of the system. In the future optical access technology plays an important role.

\section{REFERENCE}

[1] Liwei Yang. Passive optical network and the study of chaotic spread spectrum code division multiple access to the light. Beijing University of Posts and Telecommunications. 2009

[2] Jianbo Chen. OCDMA-PON system and its key technology research. Beijing University of Posts and Telecommunications .2011

[3] Di Zhang. Based on two-dimensional double carrier frequency hopping OCDMA system design and its confidentiality. Central China normal university. 2013

[4] Chuanqi Li, Xu Zhou, Biao Song. A kind of passive optical division multiple access network and its performance study. Journal of Optics. 2010:660-665 
[5] Haibo Ge, Haitong Wang. Main parameters affecting OCDMA system. Optical communication Technology.2008:12-14

[6] Xiaomei Fu, Jinglong Yu, Wenrui Wang. Two-dimensional optical orthogonal code encoding optical code division multiple access system research. Journal of Optoelectronics Laser. 2005:1459-1462

[7] Yuanyuan Zhou, Yuan Zhang, Wanyi Yi. OCDMA system changing Shigemitsu are in the face, the structure of the code and the analysis of bit error rate. Journal of Nanjing information engineering university.2010:533-537 